\title{
Psychometric Properties of the Korean Version of the Clinical Language Disorder Rating Scale (CLANG)
}

\author{
Seon-Cheol Park ${ }^{1,2}$, Eun Young Jang ${ }^{3}$, Kang Uk Lee ${ }^{4}$, Jung Goo Lee ${ }^{5}$, Hwa-Young Lee ${ }^{6}$, Joonho Choi ${ }^{2,3}$ \\ 'Department of Psychiatry, Yong-In Mental Hospital, Yongin, ${ }^{2}$ Institute of Mental Health, Hanyang University, Seoul, ${ }^{3}$ Department of \\ Psychiatry, Hanyang University Guri Hospital, Guri, 'Department of Psychiatry, Kangwon University School of Medicine, Chuncheon, \\ ${ }^{5}$ Department of Psychiatry, Inje University Haeundae Paik Hospital, Busan, ${ }^{6}$ Department of Psychiatry, Soonchunhyang University Cheonan \\ Hospital, Cheonan, Korea
}

\begin{abstract}
Objective: Our study aimed to measure inter-rater and test-retest reliability, concurrent and convergent validity, and factor solutions of the Korean version of the Clinical Language Disorder Rating Scale (CLANG).

Methods: The Korean version of the CLANG for assessing thought, language, and communication, the Brief Psychiatric Rating Scale, Young Mania Rating Scale, and Calgary Depression Scale for Schizophrenia were used to evaluate language disorder, formal thought disorder, positive and negative symptoms, manic symptoms, and depressive symptoms, respectively, in 167 hospitalized patients with schizophrenia. The factor solution was obtained by the direct oblimin method. A receiver operating characteristic curve was used to find the optimal cut-off score for discriminating schizophrenia patients with and without disorganized speech.

Results: Inter-rater reliability was considered moderate (intraclass coefficient $=0.67, F=3.30, p=0.04$ ), and test-retest reliability was considered high $(r=0.94, p<0.001)$. Five factors, namely, pragmatics, disclosure, production, prosody, and association, were identified. An optimal cut-off score of 7 points with $84.5 \%$ sensitivity and $81.7 \%$ specificity was proposed for distinguishing schizophrenia patients with and without disorganized speech.

Conclusion: Our findings suggest that the Korean version of the CLANG is a promising tool for evaluating language disorder in patients with schizophrenia.
\end{abstract}

KEY WORDS: Clinical Language Disorder Rating Scale (CLANG); Disorganized speech; Formal thought disorder; Schizophrenia

\section{INTRODUCTION}

Language disorders are regarded as one of the core features of schizophrenia and have shown greater specificity for diagnosing schizophrenia than Schneiderian first-rank symptoms. ${ }^{1)} \mathrm{Crow}^{2,3)}$ proposed that, from the viewpoint of evolutionary psychiatry, genetic variation associated with schizophrenia can be related to language as a speciation event, and the nuclear symptoms of schizophrenia can be regarded as disturbances of the 'axis of indexicality' and/or extreme forms of language disorder. In addition, language disturbances have been adopted as one of the diagnostic criteria for schizophrenia in the Diagnostic and Statistical

\footnotetext{
Received: May 19, 2015 / Revised: June 24, 2015

Accepted: July 18, 2015

Address for correspondence: Joonho Choi, MD, PhD

Department of Psychiatry, Hanyang University Guri Hospital, 153 Gyeongchun-ro, Guri 11923, Korea

Tel: +82-31-560-2273, Fax: +82-31-554-2599

E-mail: jchoi@hanyang.ac.kr
}

Manual of Mental Disorders (DSM). With the gradually increasing empirical trend in consecutive versions of the DSM, the description of language disorders has changed from incoherence or marked loosening of associations to disorganized speech. ${ }^{4-6)}$ Andreasen ${ }^{7,8)}$ developed a scale for assessing thought, language, and communication (TLC scale) to quantitatively evaluate formal though disorder (FTD), and Andreasen and Glove ${ }^{9)}$ revised the TLC scale by redefining the psychopathological terms. On the other hand, Andreasen ${ }^{10)}$ also pointed that, with the increasing empirical trend in consecutive versions of the DSM, phenomenological reduction of abnormal self-experiences and other schizophrenic Gestalt, has faded into oblivion in the diagnostic criteria. Hence, in order to complement these limitations of the TLC scale, the Thought and Language Disorder (TALD) scale, which can cover both objective and subjective FTD, has been developed. ${ }^{11)}$

However, psycholinguistic concepts were not included in any of items of the TLC scale. The Clinical Language

(a) This is an Open-Access article distributed under the terms of the Creative Commons Attribution Non-Commercial License (http://creativecommons.org/licenses/by-nc/4.0) which permits unrestricted non-commercial use, distribution, and reproduction in any medium, provided the original work is properly cited. 
Disorder Rating Scale (CLANG) has been developed to cover language disorganization, which is a reflection of an observable expression of FTD, and its items have been constructed from the areas of phonemics, syntax, semantics, and discourse. ${ }^{12)}$ Thus, the CLANG has been used in several studies to evaluate the severity of language disorganization in patients with schizophrenia. ${ }^{1,13,14)}$ Despite the availability of the CLANG, to our knowledge, the Korean version of the CLANG has not been standardized and the diverse psychometric properties of the CLANG have not been examined. Hence we aimed to (i) assess the inter-rater and test-retest reliability and concurrent and convergent validity of the Korean version of the CLANG, (ii) extract its factor structure and characterize each of the factors, and (iii) propose the optimal cut-off value (together with its sensitivity and specificity) for the presence of disorganized speech in Korean patients with schizophrenia.

\section{METHODS}

\section{Study Overview}

As described elsewhere, ${ }^{15)}$ the registry of speech samples of Korean patients with schizophrenia was used to identify the psychometric properties of the Korean version of the CLANG. Only the Korean version of the CLANG was used in the present work. Our inclusion criteria were (i) diagnosis of schizophrenia with DSM 5th edition [DSM-5], ${ }^{6}$ (ii) age $\geq 18$ years and $\leq 65$ years, (iii) psychiatric admission of 2 weeks or more, and (iv) educational attainment of elementary school or more. Conversely, our exclusion criteria were (i) comorbid organic mental disorder, intellectual disability, or alcohol/substance use disorders (with DSM-5); ${ }^{6}$ (ii) comorbid seizure disorder or other neurological diseases; and (iii) comorbid severe medical or surgical diseases. Overall 167 inpatients with schizophrenia were recruited at Yong-In Mental Hospital and National Chuncheon Hospital, Korea, from January 2014 to June 2014. The institutional review board of Yong-In Mental Hospital (receipt number 2013-49) approved our study protocol. Before starting the study, informed consent was obtained from all the study subjects.

Before initiation of the study, a one-day workshop for training in the CLANG and other psychometric scales was attended by 2 psychiatrist (SCP, JC) and 4 psychiatric residents (HJY, SMH, BK, JMY). Manual review, rating of audiotape-recorded CLANG interviews, and discussion were included in the workshop. Three (SCP, JC, HYL) of the 6 raters, chosen at random, assessed the severity of language disorganization from audiotape-recorded inter- views using the CLANG. Inter-rater reliability was evaluated from 4 independent ratings of the same interviews. During the interviews, spontaneous speech about a neutral theme, an answer to an open question, or repetition of a brief story of neutral content was used to elicit a speech sample under standardized conditions. The mean time to complete the questions for the CLANG and other psychometric scales including the TLC scale, Brief Psychiatric Rating Scale (BPRS), ${ }^{16)}$ Young Mania Rating Scale (YMRS) ${ }^{17)}$ Calgary Depression Scale for Schizophrenia (CDSS), ${ }^{18)}$ and disorganized speech domain of Clinician Rated Dimensions of Psychosis Symptom Severity (CRDPSS $)^{19)}$ was about 80 minutes. The test-retest reliability of the CLANG was evaluated from 3-to-4-week rerating data in a non-random sample of 148 of our study subjects.

\section{The CLANG and Its Forward and Backward Translation}

As shown in Table 1, the CLANG consisted of 17 items, all of which were rated on a Likert scale from 0 (normal) to 3 (severe). Before starting the translation process, we received permission from Dr. Eric Y.H. Chen to develop the Korean version of the CLANG and use it in a psychometric validation study. Consistent with the guidelines for developing other language versions of the scale, ${ }^{20)}$ its forward and backward translation was performed. A beta version of the Korean translation was made from separate English-to-Korean translations by two independent psychiatrists (SCP, JC). A consensus version, based on consensus between the two psychiatrists, was backwards translated into English by a bilingual clinical psychologist (JJ). By carefully comparing the original version and the backwards translation version of the CLANG, with some revisions, the final Korean version of CLANG used in this study was developed.

\section{Other Psychometric Scales}

The TLC scale, ${ }^{7-9)}$ BPRS,${ }^{16)}$ YMRS $,{ }^{17)} \mathrm{CDSS},{ }^{18)}$ and disorganized speech domain of the CRDPSS ${ }^{19)}$ were used to evaluate FTD, overall symptoms, manic symptoms, depressive symptoms, and presence of disorganized speech, respectively. The TLC scale, BPRS, YMRS, and CDSS had been formally translated into Korean and standardized in Korean subjects, ${ }^{15,21-23)}$ whereas the disorganized speech domain of the CRDPSS had not been formally translated into Korean. Hence, we used the English version of disorganized speech of the CRDPSS in our study. Half the items on the 18-item TLC scale were rated from 0 (absent) to 4 (extreme) and the other half from 0 (absent) to 3 (severe). For the 18-item BPRS, all items were rated from 
Table 1. Mean item scores on the CLANG

\begin{tabular}{|c|c|c|c|c|c|}
\hline No & Item & Mean & Standard deviation & Skewness & Kurtosis \\
\hline 1. & Excess phonetic association & 0.10 & 0.03 & 3.92 & 15.32 \\
\hline 2 & Abnormal syntax & 0.53 & 0.07 & 1.54 & 1.28 \\
\hline 3 & Excess syntactic constrainns & 0.32 & 0.05 & 2.25 & 5.47 \\
\hline 4 & Lack of semantic association & 0.74 & 0.09 & 1.16 & -0.19 \\
\hline 5 & Referential failures & 0.12 & 0.04 & 4.68 & 22.81 \\
\hline 6 & Disclosure failure & 0.99 & 0.08 & 0.62 & -1.02 \\
\hline 7 & Excess details & 0.64 & 0.06 & 1.09 & 0.37 \\
\hline 8 & Lack of details & 1.00 & 0.80 & 0.64 & -0.81 \\
\hline 9 & Aprosodic speech & 0.59 & 0.07 & 1.42 & 0.95 \\
\hline 10 & Abnormal prosody & 0.17 & 0.04 & 3.62 & 13.6 \\
\hline 11 & Pragmatics disorder & 0.46 & 0.06 & 1.71 & 1.88 \\
\hline 12 & Dysfluency & 0.40 & 0.05 & 1.69 & 2.30 \\
\hline 13 & Dysarthria & 0.62 & 0.07 & 1.38 & 0.68 \\
\hline 14 & Poverty of speech & 0.88 & 0.08 & 0.92 & -0.57 \\
\hline 15 & Pressure of speech & 0.36 & 0.05 & 1.98 & 3.45 \\
\hline 16 & Neologisms & 0.13 & 0.04 & 4.29 & 19.17 \\
\hline \multirow[t]{2}{*}{17} & Paraphasic error & 0.26 & 0.05 & 2.74 & 6.69 \\
\hline & Total score & 8.31 & 6.90 & 0.93 & 0.26 \\
\hline
\end{tabular}

CLANG, Clinical Language Disorder Rating Scale.

1 (absence) to 7 (extremely severe). For the 11-item YMRS, 4 items (irritability, speech, content, and disruptiveaggressive behaviors) were rated from 0 (normal) to 8 (severe) and the remaining items from 0 (normal) to 4 (severe). For the CDSS, all items were rated from 0 (normal) to 3 (severe). The disorganized speech domain of the CRDPSS was transformed to a dummy variable, since its presence was defined as a given score of 2-4 (mild-severe) and its absence a score of 0-1 (not present-equivocal).

As proposed by Park et al., ${ }^{15)}$ the TLC scale was divided into 3 subscales, namely, fluent disorganization, speech emptiness, and speech peculiarity. According to the proposal of Lachar et al., ${ }^{24)} 2$ subscales consisting of positive symptoms (conceptual disorganization, suspiciousness, hallucinatory behavior, unusual thought content, and disorientation items) and negative symptoms (emotional withdrawal, motor retardation, and blunted affect items) were used in the BPRS. In addition, according to Lahti et $a l .{ }^{25)}$ disorganization was defined with score on the item 4 in the BPRS.

\section{Statistical Analyses}

The inter-rater reliability for the four independent ratings was evaluated by the intraclass correlation coefficient (ICC), and the test-retest reliability by Pearson's correlation coefficient $(r)$.

The direct oblimin method with maximum likelihood extraction was used to perform an exploratory factor analysis (EFA) of the Korean version of CLANG, since inter-relations among the items could not be excluded. In ac- cordance with Kaiser's rule (eigenvalue $>1$ ), ${ }^{26)}$ the appropriate number of factor solutions was estimated on a scree plot. The internal consistency of each of the factors was evaluated with Cronbach's $\alpha$, and the concurrent and convergent validity of each of the factors was evaluated with Pearson's correlation coefficients ( $r s)$. Statistical significance was set at $p<0.05$ (two-tailed) for all tests.

In the Korean version of CLANG, the optimal cut-off value, which discriminated schizophrenia patients with disorganized speech from those without disorganized speech, was identified by receiver operating characteristic (ROC) curve analysis. As described elsewhere, ${ }^{27,28)}$ the ROC curve model was developed from signal-detection theory and has been widely used in previous biological studies. Since the optimal cut-off score was defined as the score with the lowest numbers of false positives and false negatives, in calculating overall predictor performance, the sensitivity/specificity pair was defined. All statistical analyses were performed with PASW Statistics software ver. 18.0 for Windows (IBM Co., Armonk, NY, USA).

\section{RESULTS}

\section{Study Subjects and Descriptive Characteristics}

As described elsewhere, ${ }^{15)}$ the mean age of the 167 study subjects was 46.5 years (standard deviation $[\mathrm{SD}]=11.2$ ). The mean duration of illness was 20.9 years $(\mathrm{SD}=10.3)$. Most were unmarried $(\mathrm{n}=129 ; 77.2 \%)$, with educational attainment of below high school graduate $(n=108 ; 64.7 \%)$, religiously affiliated $(\mathrm{n}=99 ; 59.3 \%)$, and recruited from 
Yong-In Mental Hospital ( $\mathrm{n}=145 ; 86.8 \%)$. About half were men $(\mathrm{n}=86 ; 51.5 \%)$. Average chlorpromazineequivalents of the prescribed antipsychotic medications was $921.1 \mathrm{mg}(\mathrm{SD}=952.0)$. The mean total scores on the TLC scale, BPRS, YMRS, CDSS were 11.0 (SD=9.5), 40.1 $(\mathrm{SD}=12.3), 7.3(\mathrm{SD}=6.9)$, and $1.5(\mathrm{SD}=2.5)$, respectively.

As shown in Table 1, the average score on lack of details was more than 1 point, whereas the average scores on the other items were less than 1 point. Based on Bulmer's criteria, ${ }^{29)} 14$ items had strongly positive skewness $(>+1)$ and the remaining 3 items had moderately positive skewness (between +0.05 and +1 ). There were no approximately symmetric and normally distributed items.

\section{Inter-Rater and Test-Retest Reliability}

The inter-rater reliability of the four independent ratings of the same speech samples was 0.67 ( $F=3.30$, $p=0.04$ ) of the ICC and this was considered a moderately favorable level. In addition, test-retest reliability for 3 to 4 weeks was $r=0.94(p<0.001)$ and considered a highly favorable level.

\section{Exploratory Factor Analysis (EFA)}

The number of subjects in our study was greater than the recommended minimum sample size for performing EFA $(\mathrm{n}>150) \cdot{ }^{30)}$ Barlett's test for specificity was significant $\left(\chi^{2}[167]=158.4\right.$, degree of freedom $=61, p<$ 0.01 ). Based on these indicators, EFA included all the items of the Korean version of the CLANG. Since the eigenvalues greater than 1 were limited to the first to the fifth components in Figure 1, a five-factor solution was extracted based on Kaiser's rule. ${ }^{26)}$ The loadings and Cronbach's $\alpha$ values of the five-factor solution are pre-

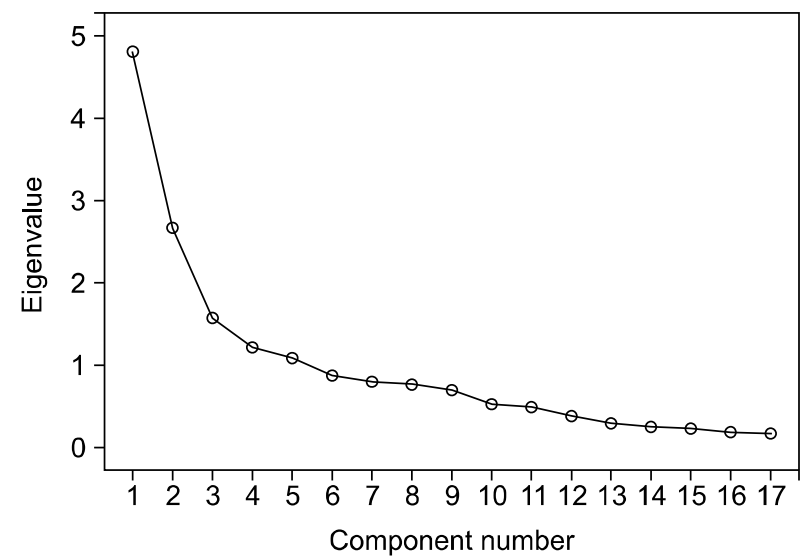

Fig. 1. Scree plot of the factor eigenvalues of the Korean version of the Clinical Language Disorder Rating Scale.

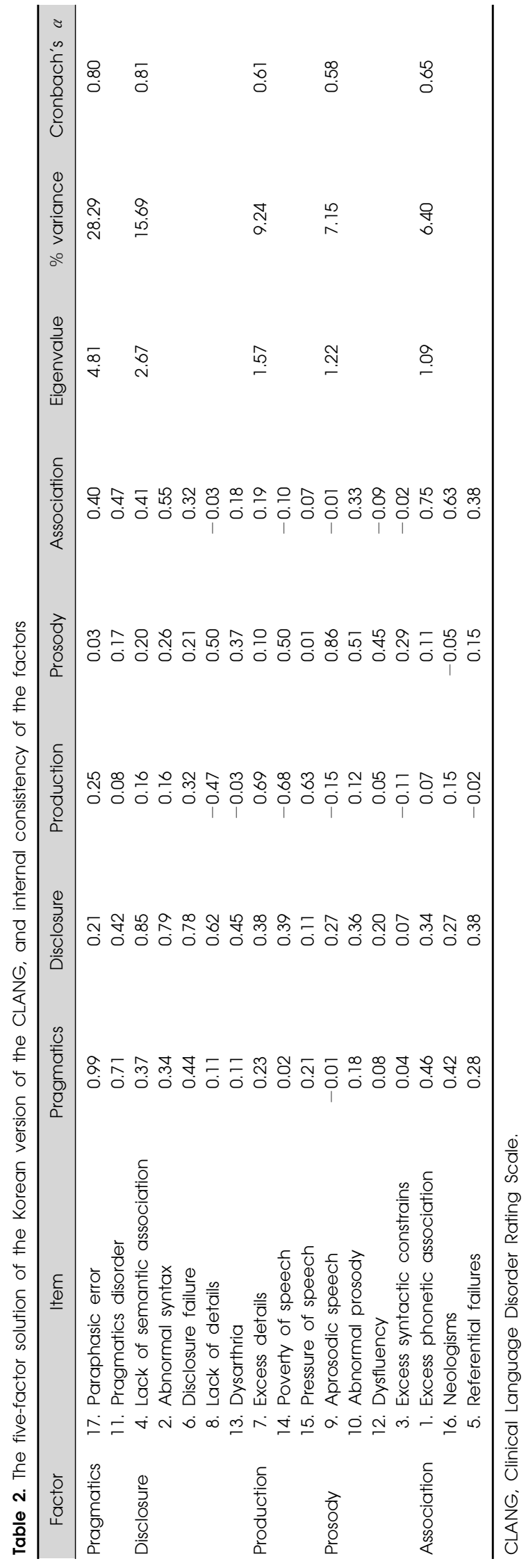


sented in Table 2. The first factor consisting of the paraphasic error and pragmatic disorder items, is designated 'pragmatics.' The second factor consisting of lack of semantic association, abnormal syntax, disclosure failure, lack of details, and dysarthria, is named 'disclosure.' The third factor consisting of excess details, poverty of speech (negative weight), and pressure of speech, is referred to as 'production.' The fourth factor consisting of aprosodic speech, abnormal prosody, dysfluency, and excess syntactic constraints, is named 'prosody.' Although the factor loading of the excess syntactic constraints was less than 0.40 , because of its conceptual similarity it was aggregated to the prosody factor. The fifth factor consisted of excess phonetic association, neologisms, and referential failures, and was named 'association.' Although the factor loading of referential failure was less than 0.40 , because of its conceptual similarity it was aggregated to the association factor. The Cronbach's $\alpha$ values of the five factors ranged from 0.58 to 0.81 . In terms of internal consistency, two factors were considered $\operatorname{good}(0.70 \leq \alpha<0.90)$, two were acceptable $(0.60 \leq \alpha<0.70)$, and one was poor $(0.50 \leq \alpha<0.60)$.

As shown in Table 3, correlation coefficients among the five factors ranged from -0.14 to 0.65 . Significant correlations were found between the following factors: pragmatics and disclosure $(r=0.65, p<0.001)$, pragmatics and prosody $(r=0.21, p<0.001)$, pragmatics and association $(r=0.60, p<0.001)$, disclosure and prosody $(r=0.34, p<$
$0.001)$, and disclosure and association $(r=0.38, p<0.001)$.

\section{Concurrent and Convergent Validity}

As shown in Table 4, the correlation coefficients among the factors of the CLANG and TLC scale ranged from -0.35 to 0.82 , and were significant, except for that between production and speech peculiarity $(r=-0.03$, $p=0.73$ ). The correlation coefficients between the factors of CLANG and the positive and negative symptom subscales and disorganization item of BPRS ranged from -0.42 to 0.71 , and were significant, except for that $(r=0.15, p=0.06)$ between association and negative symptoms. Pragmatics $(r=0.40, p<0.001)$, disclosure $(r=0.42, p<0.001)$, and production $(r=0.42, p<0.01)$ were significantly correlated with YMRS, whereas prosody $(r=0.01, p=0.09)$ and association $(r=0.05, p=0.52)$ were not. In addition, there were no significant correlations between CDSS and the factors of the CLANG.

\section{Receiver Operating Characteristic (ROC) Curve Analysis}

As shown in Figure 2, ROC curve analysis showed that the CLANG clearly distinguished between schizophrenia patients with and without disorganized speech (area under the curve $[\mathrm{AUC}]=0.89, p<0.01)$. To distinguish schizophrenia patients with and without disorganized speech, the optimal cut-off total score on the CLANG was identified as 7 points with $84.5 \%$ sensitivity and $81.7 \%$ specificity, respectively.

Table 3. Correlations between factors in the five-factor solution of the Korean version of the CLANG

\begin{tabular}{lcccc}
\hline Factor & Pragmatics & Disclosure & Production & Prosody \\
\hline Pragmatics & 1.00 & & & \\
Disclosure & $0.65^{* *}$ & 1.00 & 1.00 & -0.14 \\
Production & 0.15 & 0.09 & 0.11 & 1.00 \\
Prosody & $0.21^{* *}$ & $0.34^{* *}$ & $0.38^{* *}$ & 0.10 \\
Association & $0.60^{* *}$ & 0.00 & 1.00 \\
\hline
\end{tabular}

CLANG, Clinical Language Disorder Rating Scale.

${ }^{*} p<0.05,{ }^{* *} p<0.01$.

Table 4. Correlations between the Korean version of the CLANG and other psychometric scales

\begin{tabular}{lcccc}
\hline \multicolumn{1}{c}{ Scale } & Pragmatics & Disclosure & Production & Prosody \\
\hline Fluent disorganization (TLC scale) & $0.82^{* *}$ & $0.74^{* *}$ & $0.33^{* *}$ & $0.19^{* *}$ \\
Speech emptiness (TLC scale) & $0.40^{* *}$ & $0.49^{* *}$ & $-0.35^{* *}$ & $0.48^{* *}$ \\
Speech peculiarity (TLC scale) & $0.38^{* *}$ & $0.34^{* *}$ & -0.03 & $0.53^{* *}$ \\
Positive symptom (BPRS) & $0.66^{* *}$ & $0.62^{* *}$ & $0.24^{* *}$ & $0.17^{*}$ \\
Negative symptom (BPRS) & $0.37^{* *}$ & $0.38^{* *}$ & $-0.42^{* *}$ & $0.20^{*}$ \\
Disorganization (BPRS) & $0.64^{* *}$ & $0.71^{* *}$ & $0.19^{* *}$ & $0.45^{* *}$ \\
YMRS & $0.40^{* *}$ & $0.42^{* *}$ & $0.42^{* *}$ & 0.01 \\
CDSS & $-0.20^{*}$ & -0.02 & -0.04 & $0.41^{* *}$ \\
\hline
\end{tabular}

CLANG, Clinical Language Disorder Rating Scale; TLC scale, Scale for the Assessment of Thought, Language and Communication; BPRS, Brief Psychiatric Rating Scale; YMRS, Young Mania Rating Scale; CDSS, Calgary Depression Scale for Schizophrenia. ${ }^{\star} p<0.05$, ${ }^{* *} p<0.01$. 


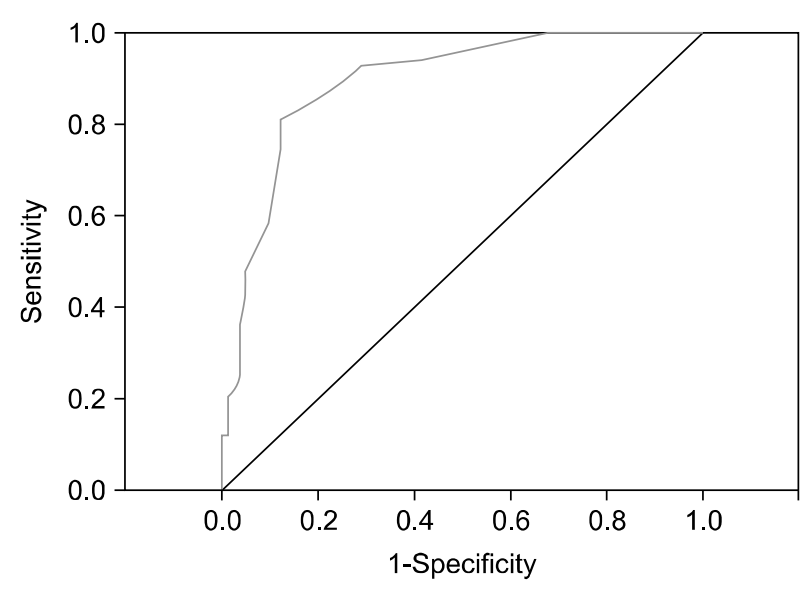

Fig. 2. Receiver operating characteristic curves for the total score on the Clinical Language Disorder Rating Scale in schizophrenia patients with and without disorganized speech.

\section{DISCUSSION}

We found that the inter-rater reliability of the Korean version of the CLANG was moderately favorable and its test-retest reliability highly favorable. A five-factor solution, which consists of pragmatics, disclosure, production, prosody, and association, was extracted from the EFA. Each of the factors has relatively selective correlations with the TLC scale, BPRS, YMRS, and CDDS. For detecting disorganized speech in patients with schizophrenia, the optimal cut-off score was 7 points with reasonable sensitivity and specificity.

Chen et al. ${ }^{12)}$ obtained a five-factor solution, namely, syntactic, semantic, production, pressure, and prosody, while Ceccherini-Nelli et al. ${ }^{13)}$ reported a three-factor solution, consisting of semantic, poverty, and excess. Some of the differences from our findings may be due to differences in the types of statistical analysis performed, namely, eigenvalues, visual inspection of scree plots, and EFA (principal component analysis with varimax rotation). The differences might also be due to variations in the number and clinical characteristics of the study subjects. However, some items of the CLANG may be present simultaneously and may share similar underlying concepts. For example, both excessive level of detail and pressure of speech may share the concept of quantitative increase of language content. In terms of the EFA of the CLANG, oblique rotation may be more valid than orthogonal rotation, although there are differences between our factor-solutions and those of Chen et al. ${ }^{12)}$ and Ceccherini- Nelli et al. ${ }^{13)}$ Hence, it appears that our study has the virtue of proposing a relatively valid factor-solution model for the CLANG.
In addition, mean duration of illness of the study subjects in our study has been greater than those in study of Chen et al. ${ }^{12)}$ Hence, we can make a speculation that greater mean duration of illness might contribute to greater severity of FTD in study subjects and association factor rather than syntactic and semantic factors might be emphasized in five-factor solution in our study. Moreover, in terms of the proposal of Fabrigar et al., ${ }^{31)}$ the sample size (30 patients with acute psychosis and 15 participants with depression symptoms) in the study of Ceccherini-Nelli et $a l .{ }^{13)}$ can be considered a small level. Hence, difference in the sample adequacy and goodness of fit of factor solution might hinder comparing the factor solutions in ours and the findings of Ceccherini-Nelli et al. ${ }^{13)}$

As shown in Table 4, the significant correlations between the five factors of CLANG and three factors of the TLC scale confirm the concurrent validity of the factors of the CLANG. These findings are partly consistent with previous findings, since Chen et al. ${ }^{12)}$ showed that the semantics factor of the CLANG was significantly correlated with total score on the TLC scale $(r=0.34, p<0.001)$ and some items of the CLANG were significantly correlated with several items on the TLC scale. Moreover, Crow ${ }^{2,3)}$ treated Schneiderian first rank symptoms (SFRS) as the extreme pole of language disorders, since the SFRS may be viewed as obliterating the boundary in communication between speaker and listener. More specifically, CeccheriniNelli et al. ${ }^{13)}$ have suggested that the SFRS can be regarded as an index of left hemisphere disconnectivity, reflecting language disturbance. In this context, CeccheriniNelli et al. ${ }^{13)}$ found significant correlations between the poverty factor of the CLANG and the passivity factor of the SFRS ( $\rho=0.61, p=0.001$ ), between total score on the CLANG and the hearing thought factor of the SFRS ( $\rho$ $=0.52, p=0.001)$, and between total scores on the CLANG and SFRS ( $\rho=0.63, p=0.001)$. However, discrimination of each of the factors of the CLANG has been little attempted.

In addition, as shown in Table 4, the significant correlations between the five factors of the CLANG, BPRS, YMRS, and CDSS support the convergent and/or divergent validity of the factors of the CLANG. This is partly consistent with Chen et al.'s findings ${ }^{12)}$ of significant correlations between the production factor of the CLANG and the negative symptom factor of the BPRS $(r=0.32, p<$ 0.001 ), between production and positive symptoms ( $r=$ $-0.25, p<0.001$, and between semantics and disorganization $(r=0.20, p=0.005)$. In our data, the significant correlations between the CLANG and YMRS can 
be attributed to the fact that the items of speech (rate and amount) and language/thought disorder were included in the YMRS. ${ }^{17)}$ Hence, the significant correlations between the CLANG and positive and negative symptoms of the BPRS partly support the suggestion made by Griesinger, ${ }^{32)}$ Kraepelin, $^{33)}$ and Bleuler ${ }^{34)}$ that FTD or disorganized speech is one of the core symptoms of schizophrenia.

To our knowledge, our study is the first to estimate inter-rater and test-retest reliabilities and a cut-off total score on the CLANG. The moderate level of inter-rater reliability may be attributed to the fact that the scores on each of the CLANG items were rated as relatively low and there is no consensus on a scoring system for each of the items. Hence, a further investigation involving a wider range of subjects and following stricter rating guidelines could increase inter-rater reliability. In addition, the interval of 3 to 4 weeks used to examine the test-retest reliability of the Korean version of the CLANG may be relatively long. Furthermore, with $84.5 \%$ sensitivity and $81.7 \%$ specificity, a cut-off total score of 7 points on the CLANG can discriminate accurately between schizophrenia patients with and without disorganized speech. Seven points on the CLANG reflects a situation in which 2 or more items are rated as severe and 4 or more as moderate.

There are several limitations to our study. First, the chlorpromazine equivalent dose of prescribed antipsychotics given to our subjects can be regard as rather high. Thus, the possibility that the prescribed antipsychotics influence the ratings on some items of the CLANG (especially, dysarthria) cannot be excluded. Second, since most of the subjects were chronically ill and hospitalized, extrapolation of our findings to other groups must be limited. Thirdly, we employed psychometric tools to assess cognitive functions in our studies. Fourthly, the disorganized speech domain of the CRDPSS was not standardized. Lastly, different statistical methods for EFA can partly interrupt comparing the factor solutions in ours and the findings of Chen et al ${ }^{12)}$ However, since there are the possible inter-relationships of all the CLANG items, oblique rotation might be more appropriate to extract factor solution of the CLANG items than orthogonal rotation in our study.

Dimensional concepts rather than categorical concepts are generally preferred for evaluating psychotic symptoms. From that viewpoint, psychosis has been regarded as cerebral disorganization reflecting the language disorders in schizophrenia. ${ }^{13)}$ Hence, despite several limitations in earlier observations, our findings have the virtue of proposing that the CLANG is one of the most promising scales for evaluating FTD or disorganized speech in patients with schizophrenia. In addition, our findings can provide the basis for evaluating language disorders and identifying their neural correlates in patients with schizophrenia. With regard to the CLANG, further study of its utility in differential diagnosing and ability to complement the subjective experiences of FTD may enlarge the usefulness of the CLANG.

\section{- Acknowledgments}

Our study was supported by the Choi Shin-Hai Neuropsychiatry Research Fund (2013) of the Korean Foundation of Neuropsychiatry Research. The funding source had no further role in the study design, in the collection, analysis and interpretation of the data, in the writing of the report or in the decision to submit the paper for publication.

We would like to thank and acknowledge the following people who contributed to the data collection and the environment for the study: Drs. Seong-Su Kim, Chang-Seok Lee, Ki Won Kim, Hyeon-Joo Yu, Seung-Min Han, Il-Joon Yi, Bomi Kim, and Jeong-Min Yu. We also would like to thank Joohwa Jeong for the backward translation of the CLANG.

\section{REFERENCES}

1. Ceccherini-Nelli A, Crow TJ. Disintegration of the components of language as the path to a revision of Bleuler's and Schneider's concepts of schizophrenia. Linguistic disturbances compared with first-rank symptoms in acute psychosis. Br J Psychiatry 2003;182:233-240.

2. Crow TJ. Is schizophrenia the price that Homo sapiens pays for language? Schizophr Res 1997;28:127-141.

3. Crow TJ. The 'big bang' theory of the origin of psychosis and the faculty of language. Schizophr Res 2008;102:31-52.

4. American Psychiatric Association. Diagnostic and Statistical Manual of Mental Disorders. 3rd ed. Washington,DC:American Psychiatric Association; 1980.

5. American Psychiatric Association. Diagnostic and Statistical Manual of Mental Disorders. 4th ed. Washington,DC:American Psychiatric Association; 1994.

6. American Psychiatric Association. Diagnostic and Statistical Manual of Mental Disorders. 5th ed. Washington,DC:American Psychiatric Association;2013.

7. Andreasen NC. Thought, language, and communication disorders. I. Clinical assessment, definition of terms, and evaluation of their reliability. Arch Gen Psychiatry 1979;36: 1315-1321.

8. Andreasen NC. Thought, language, and communication disorders. II. Diagnostic significance. Arch Gen Psychiatry 1979;36:1325-1330.

9. Andreasen NC, Grove WM. Thought, language, and communication in schizophrenia: diagnosis and prognosis. Schizophr Bull 1986;12:348-359.

10. Andreasen NC. DSM and the death of phenomenology in America: an example of unintended consequences. Schizophr Bull 2007;33:108-112.

11. Kircher T, Krug A, Stratmann M, Ghazi S, Schales C, 
Frauenheim $\mathrm{M}$, et al. A rating scale for the assessment of objective and subjective formal Thought and Language Disorder (TALD). Schizophr Res 2014;160:216-221.

12. Chen EYH, Lam LCW, Kan CS, Chan CKY, Kwok CL, Nguyen DGH, et al. Language disorganization in schizophrenia: validation and assessment with a new clinical rating instrument. Hong Kong J Psychiatry 1996;6:4-13.

13. Ceccherini-Nelli A, Turpin-Crowther K, Crow TJ. Schneider's first rank symptoms and continuous performance disturbance as indices of dysconnectivity of left- and right-hemispheric components of language in schizophrenia. Schizophr Res 2007;90:203-213.

14. Xu JQ, Hui CL, Longenecker J, Lee EH, Chang WC, Chan SK, et al. Executive function as predictors of persistent thought disorder in first-episode schizophrenia: a one-year follow-up study. Schizophr Res 2014;159:465-470.

15. Park SC, Jang EY, Lee KU, Lee K, Lee HY, Choi J. Reliability and validity of the Korean version of the scale for the assessment of thought, language, and communication. Compr Psychiatry 2015;61:122-130.

16. Overall JE, Gorham DR. The brief psychiatric rating scale. Psychol Rep 1962;10:799-812.

17. Young RC, Biggs JT, Ziegler VE, Meyer DA. A rating scale for mania: reliability, validity and sensitivity. $\mathrm{Br} J$ Psychiatry 1978;133:429-435.

18. Addington $\mathrm{D}$, Addington J, Schissel B. A depression rating scale for schizophrenics. Schizophr Res 1990;3:247-251.

19. Heckers S, Barch DM, Bustillo J, Gaebel W, Gur R, Malaspina D, et al. Structure of the psychotic disorders classification in DSM-5. Schizophr Res 2013;150:11-14.

20. Beaton DE, Bombardier C, Guillemin F, Ferraz MB. Guidelines for the process of cross-cultural adaptation of self-report measures. Spine (Phila Pa 1976) 2000;25:31863191.

21. Yi JS, Ahn YM, Shin HK, An SK, Joo YH, Kim SH, et al. Reliability and validity of the Korean version of the positive and negative syndrome scale. J Korean Neuropsychiatr Assoc 2001;40:1090-1105.

22. Jung HY, Cho HS, Joo YH, Shin HK, Yi JS, Hwang S, et al. A validation study of the Korean-version of the young mania rating scale. J Korean Neuropsychiatr Assoc 2003; 42:263-269.

23. Kim YK, Won SD, Lee KM, Choi HS, Jang HS, Lee BH, et al. A study on the reliability and validity of the Korean version of the calgary depression scale for schizophrenia (K-CDSS). J Korean Neuropsychiatr Assoc 2005;44:446-455.

24. Lachar D, Bailley SE, Rhoades HM, Espadas A, Aponte M, Cowan KA, et al. New subscales for an anchored version of the Brief Psychiatric Rating Scale: construction, reliability, and validity in acute psychiatric admissions. Psychol Assess 2001;13:384-395.

25. Lahti AC, Weiler MA, Holcomb HH, Tamminga CA, Carpenter WT, McMahon R. Correlations between $r C B F$ and symptoms in two independent cohorts of drug-free patients with schizophrenia. Neuropsychopharmacology 2006;31:221-230.

26. Kaiser HF. The application of electronic computers to factor analysis. Educ Psychol Meas 1960;20:141-151.

27. Endicott J, Spitzer RL. A diagnostic interview: the schedule for affective disorders and schizophrenia. Arch Gen Psychiatry 1978; $35: 837-844$.

28. Kraemer HC. Assessment of $2 \times 2$ associations: generalization of signal-detection methodology. Am Statistician 1998;42:37-49.

29. Bulmer MG. Principles of statistics. New York:Dover Publications; 1979.

30. Tabachnick BG, Fidell LS. Using multivariate statistics. 5th ed. Boston:Pearson Education;2007.

31. Fabrigar LR, Wegener DT, MacCallum RC, Strahan EJ. Evaluating the use of exploratory factor analysis in psychological research. Psychol Method 1999;4:272-299.

32. Griesinger W. Die Pathologie und therapie der psychischen krankheiten für ärzte und studierende [The pathology and treatment of mental illnesses for doctors and students]. 2nd ed. Stuttgart:Krabbe;1861. German.

33. Kraepelin E. Psychiatrie. 5th ed. Leipzig:Barth;1899.

34. Bleuler E. Dementia praecox oder die Gruppe der Schizophrenien [Dementia praecox or group of schizophrenia]. Leipzig:Wien;1911. German. 